\title{
NEW MATHEMATICAL MODEL FOR ANALYSING THREE-PHASE CONTROLLED RECTIFIER USING SWITCHING FUNCTIONS
}

\author{
Christos Marouchos ${ }^{*}$, M. K. Darwish ${ }^{* *}$, M. El-Habrouk ${ }^{* * *}$ \\ * Cyprus University of Technology, Limassol, Cyprus. E-mail: christos.maroucho@cut.ac.cy \\ ** Brunel University, Middlesex, UB8 3PH, U.K. E-mail: mohamed.darwish@brunel.ac.uk \\ **** Alexandria University, Alexandria, 21544, Egypt. E-mail: habrouk@gmail.com
}

\section{Abstract}

The aim of this paper is to present a set of closed form analytical equations in order to enable the computation of the three phase bridge rectifier steady state performance estimation. The proposed method presented in this paper is a fast, accurate, and effective mathematical model for analysing 3-phase full-wave controlled rectifiers. The steady state mathematical model is based on the derivation of an appropriate set of switching functions using the General Switching Matrix Circuit (GSMC) techniques. Once the switching functions are derived, the output current, input current, and output dc voltage can all be easily derived and generated from the application of this technique. The effect of overlap is accurately modelled and the distortion (notches), frequency content on the input (voltage and current), and output voltage distortion are derived. The proposed mathematical model, unlike conventional analytical methods, can be integrated in the design of active filters. Furthermore, the output voltage reduction, the rms, average and peak values of voltages and currents for the thyristors and any other semiconductor devices used are readily available for the designer by direct substitution into closed form equations without any need for the waste of time for worst case scenario simulations. This method can also be applied to other types of converters, specifically to all voltage fed power converters.

\section{Introduction}

When analysing switching power converters, it is necessary to derive expressions for voltage and current waveforms in various parts of the circuit. One method in achieving that is by using the circuit theory approach where differential equations of the system are solved either analytically or numerically with known initial conditions [1-3]. The second analysis method, using switching functions, started to develop at the early 80's and it fits well in the analysis of power converters [4-8]. States of power devices are described with switching functions [9], which get the values of ones and zeros. Furthermore, under steady state periodical operation, the switching 
functions are presented by analytical expressions in the form of the sum of infinite number of sinusoids. Although the second method could be very powerful, very little research work has taken place in this area; the time has come to reveal the true potential of the application of the switching function in power electronics.

In this paper a General Switching Matrix Circuit (GSMC) [4] is first investigated together with two matrix expressions relating input/output voltage and output/input currents by using the switching function method [9] and the analysis is presented in section 2. The 3-phase full-wave controlled rectifier mathematical model is derived from the GSMC and the matrix expressions in section 3 of the paper. In section 4 of the paper, the effect of overlap is easily modelled and these results may be used to design active filters for compensation of both line current and line voltage harmonics. This paper emphasises on the modelling of the 3-phase full-wave controlled rectifier while the active filter design is outside the scope of this paper and would be considered in future publications. Section 5 of the paper presents the comparison between the obtained results (using MathCAD) from the closed form mathematical model of sections 3 and 4 and the results from simulation (using PSPICE) and practical circuit implementations.

Two sets of switching functions, one for the thyristors and one for the overlap periods are derived. The effect of overlap on the input phase and line voltages is derived and analytical expressions for the distortion voltage are derived. These are useful in two ways: Assessing the problem of injecting voltage and current harmonics into the system and designing the PWM modulating signal for the accompanying active filters.

This new approach gives analytical expressions for both the input voltage and the input line current that is necessary for building PWM operated active filters. The output current is not approximated to a perfect $\mathrm{dc}$ and the effect of overlap on the input line current is neither ignored nor approximated to a trapezoidal shape as is the case of other analysis techniques [10]. These approximations lead to a more optimistic view of the extent of mains pollution due to a three phase ac to dc converter [10]. The analytical expression of the line current, in this paper, represents a waveform which is very close to the simulated and practical waveforms.

The analysis steps presented in this paper can be summarised to take up the following main outline:

- Development of the General Switching Matrix Circuit techniques for any general case circuit (performed in three steps)

a. Development of the $1 \times 1$ matrix for single input single output circuits

b. Development of the $3 \times 1$ matrix for the three input single output circuits 
c. Development of the $1 \times 3$ matrix for the single input three output circuits

- Application of the above techniques to the three phase AC to DC controlled rectifier circuit to generate the ideal circuit switching equations (Development of the voltage and current equations for input and output of the three phase $\mathrm{AC}$ to $\mathrm{DC}$ controlled rectifier without overlap consideration)

- Development of the switching function with consideration of the overlap period (Extended Analysis)

a. Derivation of the input voltage distortion equation with overlap considerations

b. Derivation of the output voltage equation with overlap consideration

c. Derivation of output current equation with overlap consideration

d. Derivation of input current equation with overlap consideration

- Generation (through simple substitution in the above equations) of the three phase AC to DC controlled rectifier circuit performance equations using MathCAD

- Verification of the obtained performance waveforms

a. By comparison to PSPICE simulation

b. By comparison to Practical laboratory implementation 


\section{The General Switching Matrix Circuit}

In this section the two matrices connecting input-output-voltage and output-input-current are derived for the General Switching Matrix. The General Switching Matrix will be used in the next section to derive the 3-phase rectifier circuit and the two connecting matrices will also be used to derive expressions for the output voltage and input current.

All voltage fed power electronic circuits can be derived from a GSMC. This is shown as a row $(r)$ by column (c) lines matrix; each vertical (input) line is connected to all horizontal (output) lines with $r$ switches [4]. In Fig.1, $c$ input voltage sources, $V_{l}(t), V_{2}(t), \ldots, V_{c}(t)$, are connected to $c$ vertical lines and the $r$ output voltages, $U_{1}(t), U_{2}(t), \ldots, U_{r}(t)$, are available at the $r$ horizontal lines. Every vertical line is connected to a horizontal line by an ideal switch. The output voltages drive a certain load (not shown in Fig.1) forcing the output currents $I_{\mathrm{Out}_{1}}(t), I_{\mathrm{Out}_{2}}(t)$, $\ldots, I_{\text {Out }}(t)$. These currents are reflected to the input as $I_{I N_{1}}(t), I_{I N_{2}}(t), \ldots, I_{I N_{c}}(t)$ by the action of the switches. A reference line, the neutral $(N)$, connects the input to the output directly.

Each of the $r \times c$ switches can be controlled independently in order to create an output voltage of the required frequency and magnitude. There are two restrictions [8]:

1. In a row, only one switch may be closed at any one instant of time, otherwise the voltage sources are short-circuited; i.e., no two input lines may be connected at the same time to the same output line.

2. At any times, each output line must be connected to an input line to avoid high $d v / d t$ across the switches caused by interrupting the current flow in the case of inductive loads.

The general matrix expressions relating input and output voltages, input and output currents are derived by considering $1 \times 1,3 \times 1$ and $1 \times 3$ switched circuits.

\section{The 1×1 Switching Circuit}

Consider a single switch circuit, as shown in Fig.2-a, connecting a voltage source $V_{1}(t)$ to a load; the output voltage is $U_{1}(t)$.

The simple switch circuit of Fig.2-a may also be realised by considering one vertical and one horizontal line from the GSMC as shown in Fig.2-b. In this figure, Switch $S_{11}$ connects between the input line $(r=1)$ and the output line $(c=1)$. When $S_{11}$ is closed, the output voltage, $U_{1}(t)=V_{1}(t)$. When the switch is open, the output voltage is zero. It appears from Figs.2-c, 
2-d and 2-e that the switching function is the signal which when multiplied by the input voltage gives the output voltage, therefore:

$$
U_{1}(t)=V_{1}(t) \cdot F_{11}(t)
$$

The switching function, $F_{11}(t)$, in Fig.2-c can also be represented mathematically by the following expression:

$$
F_{11}(t)=K_{o}+2 \cdot \sum_{n=1}^{\infty} K_{n} \cdot \cos (n \omega t-n \theta)
$$

where, $\quad n=$ is a positive integer number

$\omega=$ switching frequency, $\left(2 \pi f_{s}\right)$

$K_{o}=$ duty cycle of the switch

$K_{n}=\sin (n \delta) / \pi n$

$\delta \quad=$ half the $\mathrm{ON}$ period of the switch

$\theta=$ the phase angle of the switching function relative to a reference

Where, $F_{11}(t)$ is the switching function operating the switch $S_{11}$ and is defined in equation (2).

This circuit represents a typical single phase ac controller circuit. Furthermore, the same circuit of Fig. 2-a and Fig. 2-b may still represent a half wave single phase ac-dc converter circuit by simply varying the switching function from Fig. 2-c to Fig. 2-d without any need for different analysis. One may go one step further and implement the same technique on the single switch dc-dc chopper circuit by simply changing the switching function into Fig. 2-e. The technique so far proves versatile and unifies the analysis of different power electronic circuits.

\section{The $3 \times 1$ Switching Circuit}

In Fig.3-a, the voltage at the single output line, $U_{1}(t)$, is made up from the contributions of 3 input lines, $V_{1}(t), V_{2}(t)$ and $V_{3}(t)$. Therefore, this $3 \times 1$ circuit is developed to derive an expression of the output voltage in terms of the input voltages and the appropriate switching functions. In Fig.3-a, three switches in a single row connect three input lines to a single output line. Only one switch is connected to the output (horizontal line) at a time according to restriction 1. Also the output line must be connected to an input line at any time according to restriction 2. These two restrictions are demonstrated in Fig.3-b. Every input voltage source, $V_{1}(t), V_{2}(t)$ and $V_{3}(t)$, is connected to the output during the period that its switch is closed in the sequence shown in Fig.3-b. Therefore every voltage source contributes to the output voltage, $U_{1}(t)$ according to equation (1) and the total output voltage is the sum of the three contributions.

$$
U_{1}(t)=V_{1}(t) \cdot F_{11}(t)+V_{2}(t) \cdot F_{12}(t)+V_{3}(t) \cdot F_{13}(t)
$$


where, $F_{11}, F_{12}$ and $F_{13}$ are the switching functions describing the action of the witches $S_{11}, S_{12}$ and $S_{13}$ as a function of time, satisfying the condition $\left(\sum_{i=1}^{3} F_{1 i}=1\right)$ in order to conform to the two restrictions mentioned earlier.

The circuit considered, represented by the diagram of Fig. 3-a, may represent a three-phase halfwave ac-dc converter, as well as a single phase output cycloconverter with bidirectional switches by simply varying the switching functions $\left(F_{11}, F_{12}\right.$ and $\left.F_{13}\right)$

The above result may be generalised in the case of multiple rows of switches (namely three in this case) connecting the same three inputs to second and third rows outputs, $U_{2}(t)$ and $U_{3}(t)$, as follows

$$
U_{2}(t)=V_{1}(t) \cdot F_{21}(t)+V_{2}(t) \cdot F_{22}(t)+V_{3}(t) \cdot F_{23}(t)
$$

and

$$
U_{3}(t)=V_{1}(t) \cdot F_{31}(t)+V_{2}(t) \cdot F_{32}(t)+V_{3}(t) \cdot F_{33}(t)
$$

Where the switching functions satisfy the generalised conditions

$$
\sum_{i=1}^{3} F_{j i}=1 ; \text { where } j=1,2 \text { and } 3
$$

Equations (3-a), (3-b) and (3-c) can be put into matrix format and the output voltage matrix can be expressed as:

$$
\left[\begin{array}{l}
U_{1}(t) \\
U_{2}(t) \\
U_{3}(t)
\end{array}\right]=\left[\begin{array}{lll}
F_{11}(t) & F_{12}(t) & F_{13}(t) \\
F_{21}(t) & F_{22}(t) & F_{23}(t) \\
F_{31}(t) & F_{32}(t) & F_{33}(t)
\end{array}\right] \cdot\left[\begin{array}{l}
V_{1}(t) \\
V_{2}(t) \\
V_{3}(t)
\end{array}\right]
$$

The circuit herein represents a $3 \times 3$ matrix converter with bidirectional switches. By eliminating the third row in the above matrix equation, and readjusting the switching function, the resulting equations would represent a three phase full wave ac-dc converter bridge .

\section{The $1 \times 3$ Switching Circuit}

In this circuit (Fig. 4-a) the output currents are reflected to the input by the switching actions of the switches. Therefore the current at a single input line is made up from the contributions of the 3 output lines. Therefore this $1 \times 3$ circuit is developed to derive an expression of the input current in terms of the output currents and the appropriate switching functions. Consider the output lines 1 to 3 of the general circuit diagram (shown in Fig.1). These are connected to the input line 1, for a period of time during which the appropriate switches are closed as shown in Fig.4-b. The two restrictions mentioned earlier in this section still apply to this configuration. 
The current in input line 1 takes the value of the current flowing in the particular output line for the period of time during which the corresponding switch is closed.

$$
\begin{array}{lll}
I_{I N_{1}}(t)=I_{\text {Out }}(t) & \text { if } & F_{11}(t)=1 \\
I_{I N_{1}}(t)=I_{\text {Out }}(t) & \text { if } & F_{21}(t)=1 \\
I_{I N_{1}}(t)=I_{\text {Out }}(t) & \text { if } & F_{31}(t)=1
\end{array}
$$

Hence the input line current is made up from the contributions of the output lines,

$$
I_{I N_{1}}(t)=I_{\text {Out }_{1}}(t) \cdot F_{11}(t)+I_{\text {Out }_{2}}(t) \cdot F_{21}(t)+I_{\text {Out }_{3}}(t) \cdot F_{31}(t)
$$

Condition 2 stated above for the number of switches turned $\mathrm{ON}$ in the switching functions $\left(F_{11}\right.$, $F_{21}$ and $F_{31}$ ) is also satisfied when these switching functions overlap implying that more than one output line is connected to single input line. In other words $\sum_{j=1}^{3} F_{j 1} \geq 1$. This is conformal with restriction 1 stated above.

Similarly for the second and third columns of switches connecting the three output lines to the second and third input lines giving $I_{I N_{2}}(t)$ and $I_{I N_{3}}(t)$.

$$
\begin{aligned}
& I_{I N_{2}}(t)=I_{\text {Out }_{1}}(t) \cdot F_{12}(t)+I_{\text {Out }_{2}}(t) \cdot F_{22}(t)+I_{\text {Out }_{3}}(t) \cdot F_{32}(t) \\
& I_{I N_{3}}(t)=I_{\text {Out }_{1}}(t) \cdot F_{13}(t)+I_{\text {Out }_{2}}(t) \cdot F_{23}(t)+I_{\text {Out }_{3}}(t) \cdot F_{33}(t)
\end{aligned}
$$

Equations (6-a), (6-b) and (6-c) can be put into a matrix format and the input current matrix can be expressed as:

$$
\left[\begin{array}{l}
I_{I N_{1}}(t) \\
I_{I N_{2}}(t) \\
I_{I N_{3}}(t)
\end{array}\right]=\left[\begin{array}{lll}
F_{11}(t) & F_{21}(t) & F_{31}(t) \\
F_{12}(t) & F_{22}(t) & F_{32}(t) \\
F_{13}(t) & F_{23}(t) & F_{33}(t)
\end{array}\right] \cdot\left[\begin{array}{c}
I_{\text {Out }_{1}}(t) \\
I_{\text {Out }_{2}}(t) \\
I_{\text {Out }_{3}}(t)
\end{array}\right]
$$

The new generalised condition applies in this case as above

$$
\sum_{j=1}^{3} F_{j i} \geq 1 ; \text { where } i=1,2 \text { and } 3
$$

\section{The 3-Phase AC to DC Controlled Rectifier}

All voltage fed power electronic circuits can be derived from the GSMC (Fig.1). In this case, the three phase converter circuit is derived from the GSMC by considering three input lines and two output lines as shown in Fig.5-a. Furthermore, the two connecting matrices of equations (4) and (7) are employed to derive the input-output-voltage and output-input-current expressions of the three phase converter. 


\section{Input/Output Voltage and Current Equations}

The matrix expression for input and output voltages may be derived from Equation (4) and can be expressed as:

$$
\left[\begin{array}{l}
U_{1}(t) \\
U_{2}(t)
\end{array}\right]=\left[\begin{array}{lll}
F_{11}(t) & F_{12}(t) & F_{13}(t) \\
F_{21}(t) & F_{22}(t) & F_{23}(t)
\end{array}\right] \cdot\left[\begin{array}{l}
V_{1}(t) \\
V_{2}(t) \\
V_{3}(t)
\end{array}\right]
$$

Expanding equation (8) results into

$$
\begin{aligned}
& U_{1}(t)=V_{1}(t) \cdot F_{11}(t)+V_{2}(t) \cdot F_{12}(t)+V_{3}(t) \cdot F_{13}(t) \\
& U_{2}(t)=V_{1}(t) \cdot F_{21}(t)+V_{2}(t) \cdot F_{22}(t)+V_{3}(t) \cdot F_{23}(t)
\end{aligned}
$$

According to Fig.5-a,

$$
U_{\text {Out }}(t)=U_{1}(t)-U_{2}(t)
$$

Substituting equations (9-a and 9-b) into (10), yields

$$
U_{\text {Out }}(t)=V_{1}(t) \cdot\left[F_{11}(t)-F_{21}(t)\right]+V_{2}(t) \cdot\left[F_{12}(t)-F_{22}(t)\right]+V_{3}(t) \cdot\left[F_{13}(t)-F_{23}(t)\right]
$$

In the same way the input line currents in the 3-phase converter can be expressed from equation 7 as:

$$
\left[\begin{array}{l}
I_{I N_{1}}(t) \\
I_{I N_{2}}(t) \\
I_{I N_{3}}(t)
\end{array}\right]=\left[\begin{array}{ll}
F_{11}(t) & F_{21}(t) \\
F_{12}(t) & F_{22}(t) \\
F_{13}(t) & F_{23}(t)
\end{array}\right] \cdot\left[\begin{array}{l}
I_{\text {Out }_{1}}(t) \\
I_{\text {Out }_{2}}(t)
\end{array}\right]
$$

Expanding equation (12) results into

$$
\begin{aligned}
& I_{I N_{1}}(t)=I_{\text {Out }_{1}}(t) \cdot F_{11}(t)+I_{\text {Out }_{2}}(t) \cdot F_{21}(t) \\
& I_{I N_{2}}(t)=I_{\text {Out }_{1}}(t) \cdot F_{12}(t)+I_{\text {Out }_{2}}(t) \cdot F_{22}(t) \\
& I_{I N_{3}}(t)=I_{\text {Out }_{1}}(t) \cdot F_{13}(t)+I_{\text {Out }_{2}}(t) \cdot F_{23}(t)
\end{aligned}
$$

From the circuit diagram of Fig.5-a

$$
I_{\text {Out }_{1}}(t)=-I_{\text {Out }_{2}}(t)=I_{\text {Out }}(t)
$$

Substituting equation (14) into (13), yields

$$
\begin{aligned}
& I_{I N_{1}}(t)=I_{\text {Out }}(t) \cdot\left[F_{11}(t)-F_{21}(t)\right] \\
& I_{I N_{2}}(t)=I_{\text {Out }}(t) \cdot\left[F_{12}(t)-F_{22}(t)\right] \\
& I_{I N_{3}}(t)=I_{\text {Out }}(t) \cdot\left[F_{13}(t)-F_{23}(t)\right]
\end{aligned}
$$

The three phase Full Wave Thyristor rectifier shown in Fig.5-b is realised by employing thyristors to replace the ideal switches of Fig.5-a. The familiar circuit diagram of the three phase controlled rectifier circuit is shown in Fig.5-c. Equation (11) can be used to derive the output voltage and hence the output current. Equation (15) can be used to derive the input currents. 
The displacement power factor, total harmonic distortion, distortion factor, and the frequency spectrum of the input current can be extracted from the input line current expressions.

\section{The Associated Switching Functions}

It was stated in section 2 that the switching function describes the switching action of the switch and in the case of the three phase ac to dc thyristor converter it describes the switching action of the thyristors. Therefore the conduction period of a thyristor defines the on period $(2 \delta)$ of the switching function associated with the specific thyristor. A thyristor is conducting when it is forward biased and the appropriate gate requirements are satisfied. Hence the conduction period of a thyristor is a function of the triggering angle $\boldsymbol{\alpha}$, the voltage polarity of the input phase voltage connected to it and the type of the load (resistive or inductive), as well as the supply inductance. It is worthwhile to note that for continuous-conduction the conduction period of the thyristors in this circuit is $120^{\circ}$; hence $\delta_{\max }=60^{\circ}$. The switching function expression given in (2) can be generalised to every switch in the matrix as:

$$
F_{j i}(t)=K_{o}+2 \cdot \sum_{n=1}^{\infty} K_{n} \cdot \cos \left\{n \omega t-n\left[\alpha+(i-1) \frac{2 \pi}{3}+\pi(j-1)\right]\right\}
$$

Where,

$$
\begin{array}{ll}
K_{o} & =\frac{1}{3} \\
K_{n}=\frac{\sin \left(\frac{n \delta}{2}\right)}{n \pi} & \\
\mathrm{r}= & \text { number of rows }=2 \\
\mathrm{c}= & \text { number of columns }=3 \\
\mathrm{i}=1,2,3 . & \\
\mathrm{j}=1,2 . &
\end{array}
$$

The term $\left[\alpha+(i-1) \frac{2 \pi}{3}+\pi(j-1)\right]$ is the phase angle $\theta$ in equation (2) and is a function of the triggering angle ' $\boldsymbol{\alpha}$ ' and the position of the thyristor in the matrix circuit ' $i$ ' and ' $j$ '. Hence the switching functions $F_{11}, F_{12}, F_{13}, F_{21}, F_{22}$ and $F_{23}$ in Fig.5-d, can be derived from equation (16) by substituting the appropriate ' $i$ ' and ' $j$ ' values.

Equations (11) and (15) give the output voltage and input current respectively for the three phase controlled thyristor converter. The general switching function equation in (16) is applied. The 
effect of overlap is not included in these equations. This is going to be dealt with in the next section.

\section{Extended Analysis with Overlap Consideration}

The input phase voltage is distorted by the overlap. This characteristic of the rectifier is not included in expressions for the output voltage (11) and input current (15). The distorted waveforms of the actual input voltages would be required in the design of any appropriate active filters. New switching functions will be developed here to take account of the overlap.

The first overlapping period takes place at $30^{\circ}$ plus the triggering angle $\alpha$. Each overlapping period is phase displayed by $60^{\circ}$ after the first one, as marked by the letters "A" to "F" in Fig.6-a. The overlapped periods can be represented by relatively narrower switching functions $\left(F_{m}(t)\right)$, where $m$ indicates the order of the commutation and can take the number from 1 to 6 (Fig.6-b). $F_{m}(t)$ can be expressed as:

$$
F_{m}(t)=K_{s o}+2 \cdot \sum_{n=1}^{\infty} K_{s n} \cdot \cos \left\{n \omega t-n\left[\theta_{s}+\frac{\pi}{3} \cdot(m-1)\right]\right\}
$$

where,

$$
\begin{aligned}
& K_{s n}=\frac{\sin \left(\frac{n \gamma}{2}\right)}{n \pi} \\
& \left.K_{s o}=\frac{\gamma}{2 \pi} \quad \text { (duty cycle of } F_{m}(t)\right) \\
& \theta_{s}=\frac{\pi}{6}+\alpha+\frac{\gamma}{2}
\end{aligned}
$$

$\gamma$ is the overlap angle (the ON period of $F_{m}$ ).

$\omega=$ switching frequency, $\left(2 \pi f_{s}\right)$. It is also the mains frequency.

The overlap angle ' $\gamma$ ' is usually calculated by considering a perfect dc output current. In this paper ' $\gamma$ ' is calculated, as it is shown in Appendix A, by considering a finite value of the load inductance and hence more realistic calculations of the overlap periods. Fig.6-b is a display of the input phase voltages and the associated switching functions for the analysis of the three phase controlled rectifier.

\subsection{Input Voltage Distortions}

The input voltage of the 3-phase converter can be split into two sections: during the overlap period and during the period free from overlap. 
During the first commutation, marked as ' $\mathrm{A}$ ' in Fig.6-a, lines ' 3 ' and ' 1 ' are briefly short circuited and the phase voltage is reduced to $\left(V_{3}(t)+V_{1}(t)\right) / 2$. The associated switching function for overlap is given from equation (17) as $F_{l}(t)$. During the second commutation period ('B'), lines ' 3 ' and ' 2 ' are short circuited and the phase voltage is reduced to $\left(V_{3}(t)+V_{2}(t)\right) / 2$ and the associated switching function is again given from equation (17) as $F_{2}(t)$. During the third commutation period (' $\mathrm{C}$ '), lines ' 1 ' and ' 2 ' are short circuited together and the phase voltage is reduced to $\left(V_{1}(t)+V_{2}(t)\right) / 2$ and the associated switching function is again given from equation (17) as $F_{3}(t)$. In the same way the $4^{\text {th }}, 5^{\text {th }}$ and $6^{\text {th }}$ commutation instances can be derived. The overlap switching functions $F_{l}(t)$ to $F_{6}(t)$ can be used with the associated phase voltages under overlap to calculate the actual phase voltages at the input of the converter during overlap.

Hence the phase voltage to neutral during overlap can be expressed with the superscript ' $O$ ' as:

$$
\begin{aligned}
& V_{1}^{O}(t)=\left[F_{1}(t)+F_{4}(t)\right] \cdot\left(\frac{V_{1}(t)+V_{3}(t)}{2}\right)+\left[F_{3}(t)+F_{6}(t)\right] \cdot\left(\frac{V_{1}(t)+V_{2}(t)}{2}\right) \\
& V_{2}^{o}(t)=\left[F_{3}(t)+F_{6}(t)\right] \cdot\left(\frac{V_{1}(t)+V_{2}(t)}{2}\right)+\left[F_{2}(t)+F_{5}(t)\right] \cdot\left(\frac{V_{2}(t)+V_{3}(t)}{2}\right) \\
& V_{3}^{O}(t)=\left[F_{2}(t)+F_{5}(t)\right] \cdot\left(\frac{V_{2}(t)+V_{3}(t)}{2}\right)+\left[F_{1}(t)+F_{4}(t)\right] \cdot\left(\frac{V_{1}(t)+V_{3}(t)}{2}\right)
\end{aligned}
$$

During the periods in the mains cycle when there is no overlap the phase voltage to neutral appears undistorted at the input of the converter. The input voltages without the overlap effect can be derived by considering a set of switching functions which are derived by subtracting the overlap switching functions $\left(F_{1}(t), F_{2}(t), \ldots, F_{6}(t)\right)$ appropriate to each phase from ' 1 '. The voltages during the overlap-free periods are denoted by the superscript 'NO' as shown in the following equations:

$$
\begin{aligned}
& V_{1}^{N O}(t)=\left[1-F_{1}(t)-F_{3}(t)-F_{4}(t)-F_{6}(t)\right] \cdot V_{1}(t) \\
& V_{2}^{N O}(t)=\left[1-F_{2}(t)-F_{3}(t)-F_{5}(t)-F_{6}(t)\right] \cdot V_{2}(t) \\
& V_{3}^{N O}(t)=\left[1-F_{1}(t)-F_{2}(t)-F_{4}(t)-F_{5}(t)\right] \cdot V_{3}(t)
\end{aligned}
$$

Hence the phase voltages at the input of the converter are derived by adding the corresponding equations in (18) and (19). These computed voltages are denoted by the superscript ' $A$ ' as shown in equations (20) below and displayed, using MathCAD, in Fig.7-a. The converter has been also simulated using PSpice actual electronic components and the simulated results (shown in Fig. 7-b) show a very close agreement to the MathCAD results. 


$$
\begin{aligned}
V_{1}^{A}(t)= & {\left[1-F_{1}(t)-F_{3}(t)-F_{4}(t)-F_{6}(t)\right] \cdot V_{1}(t)+} \\
& +\left[F_{1}(t)+F_{4}(t)\right] \cdot\left(\frac{V_{1}(t)+V_{3}(t)}{2}\right)+\left[F_{3}(t)+F_{6}(t)\right] \cdot\left(\frac{V_{1}(t)+V_{2}(t)}{2}\right) \\
V_{2}^{A}(t)= & {\left[1-F_{2}(t)-F_{3}(t)-F_{5}(t)-F_{6}(t)\right] \cdot V_{2}(t)+} \\
& +\left[F_{3}(t)+F_{6}(t)\right] \cdot\left(\frac{V_{1}(t)+V_{2}(t)}{2}\right)+\left[F_{2}(t)+F_{5}(t)\right] \cdot\left(\frac{V_{2}(t)+V_{3}(t)}{2}\right) \\
V_{3}^{A}(t)= & {\left[1-F_{1}(t)-F_{2}(t)-F_{4}(t)-F_{5}(t)\right] \cdot V_{3}(t)+} \\
& +\left[F_{2}(t)+F_{5}(t)\right] \cdot\left(\frac{V_{2}(t)+V_{3}(t)}{2}\right)+\left[F_{1}(t)+F_{4}(t)\right] \cdot\left(\frac{V_{1}(t)+V_{3}(t)}{2}\right)
\end{aligned}
$$

The line voltages with distortion (notches) are given by:

$$
\begin{aligned}
& V_{12}{ }^{\text {ripple }}(t)=V_{1}^{O}(t)-V_{2}^{O}(t) \\
& V_{23}^{\text {ripple }}(t)=V_{2}^{O}(t)-V_{3}^{O}(t) \\
& V_{31}{ }^{\text {ripple }}(t)=V_{3}^{O}(t)-V_{1}^{O}(t)
\end{aligned}
$$

The distortion voltage is the difference between the voltages without distortion and the voltages with distortion. This is derived from equations (20) which gives the phase voltages during overlap. The voltages during overlap are subtracted from the phase voltages $\left(V_{1}(t), V_{2}(t)\right.$ and $\mathrm{V}_{3}(\mathrm{t})$ ), during the periods of the overlap. This is performed in equation (22) below, which gives the distortion voltages of the input phase voltages.

$$
\begin{aligned}
& V_{1}^{D}(t)=\left[F_{1}(t)+F_{4}(t)\right] \cdot\left(V_{1}(t)-\frac{V_{1}(t)+V_{3}(t)}{2}\right)+\left[F_{3}(t)+F_{6}(t)\right] \cdot\left(V_{1}(t)-\frac{V_{1}(t)+V_{2}(t)}{2}\right) \\
& V_{2}^{D}(t)=\left[F_{3}(t)+F_{6}(t)\right] \cdot\left(V_{2}(t)-\frac{V_{1}(t)+V_{2}(t)}{2}\right)+\left[F_{2}(t)+F_{5}(t)\right] \cdot\left(V_{2}(t)-\frac{V_{2}(t)+V_{3}(t)}{2}\right) \\
& V_{3}^{D}(t)=\left[F_{2}(t)+F_{5}(t)\right] \cdot\left(V_{3}(t)-\frac{V_{2}(t)+V_{3}(t)}{2}\right)+\left[F_{1}(t)+F_{4}(t)\right] \cdot\left(V_{3}(t)-\frac{V_{1}(t)+V_{3}(t)}{2}\right)
\end{aligned}
$$

The line distortion voltage is found from (22) as the differences of phase voltages.

$$
\begin{aligned}
& V_{12}^{D}(t)=V_{1}^{D}(t)-V_{2}^{D}(t) \\
& V_{23}^{D}(t)=V_{2}^{D}(t)-V_{3}^{D}(t) \\
& V_{31}^{D}(t)=V_{3}^{D}(t)-V_{1}^{D}(t)
\end{aligned}
$$

Fig. 8 displays the computed phase and line distortion voltages due to overlap for the circuit of Fig.5-c. Such information can be used in the design of series active filter [11]. The magnitude and phase of the phase-voltage harmonics for a supply inductance $\left(L_{s}=50 \mu \mathrm{H}\right)$ and a triggering angle $\left(\alpha=15^{\circ}\right)$, are displayed in Table 1. The results are computed based on a load resistance $\left(R_{l d}\right)$ of $8 \Omega$ and a load inductance $\left(L_{l d}\right)$ of $1 \mathrm{mH}$. The triplen harmonics are missing in consistence with the three phase configuration. Moreover, the magnitudes of the harmonics are slowly 
falling in consistence to a narrow pulse as is the case of the phase voltage distortion (Fig.8). It is worthwhile to note that a series compensator in the form of an active filter can make use of the data in Table 1 to derive its PWM modulating signal [11]. This is outside the scope of this paper.

Table 1: Magnitudes and Phase angles of the Phase-Voltage Harmonics for $L_{s}=50 \mu \mathrm{H}$ and $\alpha=15^{\circ}, L_{l d}=1 \mathrm{mH}$ and $R_{l d}=8 \Omega$

\begin{tabular}{|c|c|c|c|c|c|c|c|c|c|c|c|c|}
\hline Order & 3 & 5 & 7 & 9 & 11 & 13 & 15 & 17 & 19 & 21 & 23 & 25 \\
\hline Voltage (V) & 0 & 1.343 & 1.342 & 0 & 1.342 & 1.341 & 0 & 1.340 & 1.339 & 0 & 1.338 & 1.337 \\
\hline Phase $\left({ }^{\circ}\right)$ & 0 & $-33^{\circ}$ & 230 & 0 & $-7^{\circ}$ & $-8.5^{\circ}$ & 0 & $0.5^{\circ}$ & $-1^{\circ}$ & 0 & $5^{\circ}$ & $2.6^{\circ}$ \\
\hline
\end{tabular}

\subsection{Output Voltage}

The output voltage without overlap of the converter was given in equation (11) as the product of the input voltage and the appropriate switching functions. The output voltage with overlap can then be derived by replacing the undistorted input voltage in equation (11) with the distorted input voltage of equation (20).

$U_{\text {out }}(t)=V_{1}^{O}(t) \cdot\left[F_{11}(t)-F_{21}(t)\right]+V_{2}^{O}(t) \cdot\left[F_{12}(t)-F_{22}(t)\right]+V_{3}^{O}(t) \cdot\left[F_{13}(t)-F_{23}(t)\right]$

Equation (24) is expanded and the dc component is also derived from the expanded expression of the output voltage by setting $n=1$ in $(n-1)$ terms [9]. An equation of the output de voltage under overlap is found in Appendix B.

\subsection{Output Current}

The output voltage, $U_{\text {Out }}(t)$ is driving a current $I_{\text {Out }}(t)$ through the load impedance $\left(L_{l d}, R_{l d}\right)$. This impedance is defined as the "harmonic impedance" [9].

$$
I_{\text {Out }}{ }^{n}(t)=\frac{U_{\text {Out }}^{n}(t)}{Z[(n \pm 1) \omega] \angle \theta_{l d}[(n \pm 1) \omega]}
$$

Where, $\omega=$ switching frequency, $\left(2 \pi f_{s}\right)$. It is also the mains frequency.

$$
\begin{aligned}
& Z[(n+1) \omega]=\sqrt{{R_{l d}{ }^{2}+\left[(n+1) \omega L_{l d}\right]^{2}}} \quad \text { and } \quad \theta_{l d}[(n+1) \omega]=\tan ^{-1}\left[\frac{(n+1) \omega L_{l d}}{R_{l d}}\right] \\
& Z[(n-1) \omega]=\sqrt{{R_{l d}{ }^{2}+\left[(n-1) \omega \cdot L_{l d}\right]^{2}}} \quad \theta_{l d}[(n-1) \omega]=\tan ^{-1}\left[\frac{(n-1) \omega L_{l d}}{R_{l d}}\right] \\
& n=1,2,3, \ldots \propto
\end{aligned}
$$


An expanded expression for the output current is found in Appendix C.

\subsection{Input Current}

The input current given by equation (15) assumes zero source impedance and hence no overlap. In order to include the effect of overlap, the line current is divided into two sets of time intervals: The first set of time intervals is the periods of time between commutations. Hence thyristor switching functions (16) are modified in order to exclude the periods of the overlap. This is performed by subtracting the appropriate overlap switching functions (17) from the thyristor switching functions. With reference to Fig.6, the line current in the first line, which is unaffected by the overlap, is given by

$$
I_{I N_{1}}(t)=I_{\text {Out }}(t) \cdot\left[\left(F_{11}(t)-F_{1}(t)\right)-\left(F_{21}(t)-F_{4}(t)\right)\right]
$$

The second set of time intervals in the first line is the overlap periods which are four per phase, as shown in Fig.6-a points A, C, D and F. The line current during the overlap is represented by a transient current component where a pulse of the line voltage is applied to the source impedances of the short circuited lines for a period equal to $\gamma$ (the overlap period). For the first commutation period (point A in Fig.6-a), the first and third lines are short circuited, allowing a transient component of a current, $I_{L}{ }^{S C}(t)$ [Appendix A]. The part of the transient current which is of interest is during the overlap, giving the rising edge of the first line current as

$$
I_{1 P O S}^{\text {transient-rising }}=F_{1}(t) \cdot I_{L}^{S C}(t)
$$

Equation 27 gives the rising edge of the first line current. In order to derive the equation for the falling edge (which occurs $120^{\circ}$ later) the third commutation, denoted by ' $\mathrm{C}$ ' in Fig.6-a, must be considered. During that commutation the first and the second lines are short circuited. The current is falling in the first line and is rising in the second line in a manner indicated by equation (A3) because the parameters of the circuit are the same. Equation (27) though has to be shifted in time by $T_{3}$ seconds (or $120^{\circ}$ ) to the instant where the $3^{\text {rd }}$ commutation starts (point C) in order to give the rising edge in the second line. The associated switching function is $F_{3}(t)$ as shown in Fig.6-b.

$$
I_{2 P O S}^{\text {transient-rising }}=F_{3}(t) \cdot\left[I_{L}^{S C}\left(t-T_{3}\right)\right]
$$

Equation (28) gives the transient of the rising edge of the current in the second line. At the same time (during commutation at point $\mathrm{C}$ ) the current in the first line is falling and in accordance with Kirchoff's current law, this current is the difference between the output current and the current in the second line.

$$
I_{1 P O S}^{\text {transient-falling }}(t)=F_{3}(t) \cdot\left\{I_{\text {Out }}(t)-\left[I_{L}^{S C}\left(t-T_{3}\right)\right]\right\}
$$


Equation (29) gives the falling edge of the positive pulse of the line current in the first line.

The negative pulse of the current in the first line starts at the $4^{\text {th }}$ commutation (point D in Fig.6b) and ends at the beginning of the $6^{\text {th }}$ commutation (point F). Both the rising and falling edges of the positive pulse, equations (27) and (29) are shifted by $180^{\circ}$ or $\mathrm{T}_{4}$ seconds and $240^{\circ}$ or $\mathrm{T}_{6}$ seconds, respectively. For the rising edge of the negative pulse the transient of equation (27) is shifted by $\mathrm{T}_{4}$ seconds

$$
I_{1 N E C}^{\text {transient-rising }}=F_{4}(t) \cdot\left[I_{L}^{S C}\left(t-T_{4}\right)\right]
$$

For the falling edge of the negative pulse the transient of equation (27) is shifted by $\mathrm{T}_{6}$ seconds

$$
I_{1 N E C}^{\text {transient-falling }}(t)=F_{6}(t) \cdot\left\{I_{\text {Out }}(t)-\left[I_{L}^{S C}\left(t-T_{6}\right)\right]\right\}
$$

Finally, the current of the first line is given from Expressions 26, 27, 29, 30 and 31 as

$$
I_{1}(t)=I_{1 N_{1}}(t)+I_{1 P O S}^{\text {transient-rising }}(t)+I_{1 P O S}^{\text {transient-falling }}(t)-I_{1 N E C}^{\text {transient-rising }}(t)-I_{1 N E C}^{\text {transient-falling }}(t)
$$

The order, magnitude and phase of the individual line current harmonics including the fundamental can be directly derived from equation (32). It is worthwhile to note that an active filter compensator can make use of this data to derive its PWM modulating signal.

\subsection{Summary}

By employing the switching function technique, outlined in this paper, a number of closed form analytical model equations are derived describing the steady state performance of the three phase controlled rectifier. The input voltage distortion is expressed in equations (20) and (23), the output voltage in equation (24), output current in equation (25) and the input current in equation (32) by considering the effect of overlap. MathCAD is used to display these voltage and current waveforms.

\section{Conformity to Simulation and Practical Results}

In order to ensure that the proposed technique of General Switching Matrix Circuit (GSMC) with the analytical expressions presented in this paper (refer to section 4.5) is valid and conforms to reality, these proposed equations are used in MathCAD and simple direct substitution (without recurrence to any integration used in simulation techniques) is used in the above equations (for input and output voltages and currents) to generate the steady state performance waveforms characteristic to the three phase controlled rectifier circuit. The resulting computed waveforms 
are plotted and compared to both ordinary simulation technique and practical implementation results for comparison and to prove the effectiveness of the proposed technique.

\subsection{Conformity to Simulation Results}

Fig.9-a displays the first line (phase a) input current computed from the above equations using MathCADd. It is a more accurate representation of the line current with overlap than the conventionally used trapezoidal approximation normally employed in ordinary simulations [10]. The test conditions for this case are as follows:

$$
\mathrm{V}_{\text {peak }}=380 \mathrm{~V} \text { line to line, } \mathrm{R}_{\text {load dc side }}=3 \Omega, \mathrm{L}_{\text {load dc side }}=40 \mathrm{mH}, \mathrm{L}_{\text {supply ac side }}=50 \mu \mathrm{H}
$$

The thyristor converter circuit is triggered at a delay angle of $\alpha=18^{\circ}$.

These computed results of Fig.9-a are compared to ordinary simulation results (using differential equations solution) from PSPICE. PSPICE results, based on actual electronic component simulation and shown in Fig.9-b, illustrate an exact simulated input line current waveform conformal to that of Fig.9-a.

\subsection{Conformity to Practical Results}

Furthermore, an experimental setup was implemented in order to compare the waveforms obtained from laboratory practical results of a three phase rectifier circuit (of Fig.5-c) with the waveforms obtained by direct substitution from the proposed analytical results. The same conditions applied to the mathematical equations of the proposed computational technique are again applied to the practical circuit.

The test results depicted in this section have the following parameters:

$$
\mathrm{V}_{\mathrm{rms}}=380 \mathrm{~V} \text { line to line, } \mathrm{R}_{\text {load dc side }}=32 \Omega, \mathrm{L}_{\text {load dc side }}=180 \mathrm{mH}, \mathrm{L}_{\text {supply ac side }}=1 \mathrm{mH}
$$

The thyristor converter circuit is triggered at a delay angle of $\alpha=7^{\circ}$.

Fig.10 shows a comparison of the practical results (Fig.10-a) and the theoretical results using MathCAD (Fig.10-b). In both figures the phase voltage and line current are displayed for $\alpha=7^{\circ}$ (note the $30^{\circ}$ shift between the first line current and the beginning of the first phase voltage). Considering that in the proposed switching function technique the thyristors are considered as ideal switches, it is then clear that there exists a close agreement between the practical results and the theoretical results using MathCAD.

The voltage and current measurements, of Fig.10-a, were performed using voltage and current Hall Effect Transducers with output amplifiers, respectively. Their respective gains were 
220Volts/Volt-Measured and 0.4Amp/Volt-Measured. Please note that the datum of each of the two waveforms shown in Fig.10-a are located at the channel marker points (1 and 2) situated at the left side of the figure.

An expansion of the first commutation period is illustrated in Fig.11-a (practical implementation) and in Fig.11-b (MathCAD computations). Again a very close agreement is observed in these two figures. In the full range of triggering angles the maximum error observed between the practical and simulated results was $\pm 2.5 \%$.

\section{Conclusions}

This paper presents a fast, accurate, and effective steady state mathematical model for analysing 3-phase full-wave controlled rectifiers. Such an approach enables the assessment of the problem of injecting voltage and current harmonics into the power system and designing the PWM modulating signal for the accompanying active filters. The proposed technique cancels the requirements for the solution of differential equations in order to assess the system performance under various control conditions. This in effect will greatly reduce the requirement for simulation times since the problem reduces merely to substituting in analytical equations. This is outside the scope of this paper and would be considered in future publications.

The mathematical model is based on the derivation of an appropriate set of switching functions. Once the switching functions are derived, the output current, input current, input voltage distortion and output de voltage can all be easily derived and generated from the application of this technique. The effect of overlap is introduced and expressions showing the currents during this overlap have been incorporated in the expression of the line current. Furthermore, the distortion (notches), frequency content of the input voltage, input and output currents and voltage distortion are derived in this paper. The voltages and currents (rms, average and peak values) of the inputs and outputs as well as voltages and currents of the thyristors can also be derived. The required mathematical expressions and derivations of the closed form analytical equations have been presented during the course of the paper and it is clear that the same technique may be applied to any type of voltage fed converters. The resulting computations are shown to be in great accordance with the simulated results from PSPICE as well the practical results, which confirms the validity and viability of the proposed technique.

\section{References}

[1] Bose B.K.:"Modern Power Electronics and AC Drives", Prentice Hall PTR, 2002.

[2] Skvarenina T.L.:"Power Electronics Handbook", CRC Press, 2002. 
[3] Shaffer, R.: "Fundamentals of Power Electronics with Matlab", Charles River Media, 2007.

[4] Wood P.: "Switching power converters", Van Nostrand Reinhold Company, New York, 1981.

[5] Marouchos C. PhD thesis: "Switched Capacitor circuits for reactive power generation", Brunel University ,1982.

[6] Byoung-Kuk Lee: "A simplified function simulation model for three-phase voltage-source inverter using switching function concept”, IEEE Transactions in Industrial Electronics, April 2001, 48(2), pp. 309-317.

[7] Marouchos, C., Darwish, M. K., and El-Habrouk, M.: "Variable VAR Compensator Circuits", IEE Proc.-Electr. Power Appl., Vol. 153, No. 5, September 2006, pp. 682-690.

[8] A. Alesina, M.G.B Venturi: "Solid-State Power Conversion: A Fourier Analysis Approach to Generalised Transformers Synthesis", IEEE Transactions on Circuits and system Analysis, , April 1981, Vol. 28, No.4, pp. 319-331.

[9] Marouchos, CC : "The switching Function: Analysis of Power Electronic Circuits", IEE, London,2006.

[10] MOHAN, N., UNDERLAND, T.M and ROBBINS, W.P“: "Power Electronics", John Willey and Sons second edition.,1995 , ISBN-0-471-58408-8.

[11] Ribeiro, E.R.; Barbi, I.: "Harmonic Voltage Reduction Using a Series Active Filter Under Different Load Conditions", IEEE Transactions on Power Electronics, Volume 21, Issue 5, Sept. 2006, pp. $1394-1402$. 


\section{Appendix A}

\section{Calculation of the overlap angle $\gamma$}

The overlap angle $\gamma$ is usually calculated from an expression [10] which assumes a perfect dc output current and is usually larger than the real duration of the overlap. This paper deals with the general case of non-perfect dc current at the output. A more accurate calculation of $\gamma$ is derived here by considering the transient current $\left(I_{L}^{S C}(t)\right)$ during overlap. When this transient current reaches the magnitude of the output current $\left(I_{\text {OUT }}(t)\right)$ (Fig. A), the overlap ceases. The duration of the transient current must be chosen to be larger than the expected value of $\gamma$ in order for the above to be valid. The duration of the transient current is dictated by the ON period of the switching function chosen to derive the transient current.

Henceforth, a general equation of the transient current $\left(I_{L}^{S C}(t)\right)$ is derived in the next few lines using the switching function. The time taken by this current to reach the output current $\left(I_{\text {OUT }}(t)\right)$ is the overlap period $\gamma\left(T_{E}\right)$.

The value of $\gamma$ derived from the textbook formula [10] is pessimistically large and is a good candidate for a worst case scenario. The switching functions describing the instances of overlaps at which line 1 is taking over from conducting line 3 are $F_{1}(t)$ and $F_{4}(t)$ as shown in Fig.6-b. The new switching function, $F_{O T}(t)$, is derived as the summation of the two switching functions and is given by:

$$
\begin{aligned}
& F_{O T}(t)=K_{O T}+2 \sum_{n=1}^{\infty} K_{T n} \cos \left\{2 n \omega t-2 n \theta_{s}\right\} \\
& K_{T n}=\frac{\sin (n \gamma)}{n \pi} \\
& K_{O T}=\frac{\gamma}{\pi}
\end{aligned}
$$

Where $\gamma$ is a value of the overlap period derived assuming a perfect dc [10]. It is chosen because it yields a larger value than the true $\gamma$.

During this overlap at the point denoted by 'A' (line 3 to line 1, Fig.6-a) a pulse of the line voltage $\mathrm{V}_{31}(\mathrm{t})$ is applied to two source impedances [10].

$$
F_{O T}(t) \cdot V_{31}(t)=2 L_{s} \frac{d I_{L}^{S C}(t)}{d t}
$$

Expanding and simplifying 
$I_{L}^{S C}(t)=\mathrm{I}_{\mathrm{o}}+\frac{\mathrm{V}_{\mathrm{p}} \sqrt{3}}{2}\left[\frac{\mathrm{K}_{\mathrm{oT}}}{\mathrm{X}_{\mathrm{s}}} \cos [\omega \mathrm{t}-\lambda]-\sum_{\mathrm{n}=1}^{\infty} \frac{\mathrm{K}_{\mathrm{Tn}}}{(2 n+1) X_{s}} \cos \left[(2 \mathrm{n}+1) \omega \mathrm{t}-2 \mathrm{n} \theta_{\mathrm{s}}-\lambda\right]+\sum_{\mathrm{n}=1}^{\infty} \frac{\mathrm{K}_{\mathrm{Tn}}}{(2 n-1) X_{s}} \cos \left[2(\mathrm{n}-1) \omega \mathrm{t}-2 \mathrm{n} \theta_{\mathrm{s}}+\lambda\right]\right]$

Where, $\quad \lambda=\frac{\pi}{6}$

$X_{s}=\omega L_{s} \quad\left(L_{s}\right.$ is the source inductance $)$

$I_{o}=$ Constant of integration

$K_{T n}, K_{T o}$ and $\theta_{s}$ are defined as in equation (A1).

The end of the period of the overlap is marked as $T_{I E}$ seconds (Fig.A). The magnitude of the output current at that instant $\left(I_{o}\right)$ is equal to the magnitude of the transient current $\left(I_{L}^{S C}\right)$; the transient will reach the value of the output current in $\mathrm{T}_{\mathrm{E}}$ seconds, Fig.A. Hence equating the transient current, (A3) to the output current from equation 25.

$$
I_{L}^{S C}(t)=I_{\text {out }}(t)=I_{0}
$$

The period of the overlap, $\mathrm{T}_{\mathrm{E}}$, is calculated from (A4). The overlap angle $\gamma$ is then derived as

$$
\gamma=2 \pi \mathrm{T}_{\mathrm{E}} / \mathrm{T}
$$

where, $\mathrm{T}_{\mathrm{E}}$ is the time duration of the overlap and $\mathrm{T}$ is the period of the mains 


\section{Appendix B: MathCAD Voltage Equations}

\section{Expanded Expression for output v oltage}

$$
\begin{aligned}
& \mathrm{V}_{\mathrm{oB1}}(\mathrm{t}):=2 \cdot \mathrm{V}_{\mathrm{p}} \cdot \sum_{\mathrm{n}=1}^{\mathrm{N}}\left|\sin \left(\frac{\mathrm{n} \cdot \pi}{2}\right)\right| \cdot \frac{\sin \left(\mathrm{n} \cdot \delta_{1}\right)}{\mathrm{n} \cdot \pi} \cdot \sin \left[(\mathrm{n}+1) \cdot \mathrm{w} \cdot \mathrm{t}-\mathrm{n} \cdot \theta_{13}+\frac{2 \cdot \pi}{3}\right]-2 \cdot \mathrm{V}_{\mathrm{p}} \cdot \sum_{\mathrm{n}=1}^{\mathrm{N}}\left|\sin \left(\frac{\mathrm{n} \cdot \pi}{2}\right)\right| \cdot \frac{\sin \left(\mathrm{n} \cdot \delta_{1}\right)}{\mathrm{n} \cdot \pi} \cdot \sin \left[(\mathrm{n}-1) \cdot \mathrm{w} \cdot \mathrm{t}-\mathrm{n} \cdot \theta_{13}-\frac{2 \cdot \pi}{3}\right] \\
& \mathrm{V}_{\mathrm{oB} 2}(\mathrm{t}):=2 \cdot \mathrm{V}_{\mathrm{p} 1} \cdot \sum_{\mathrm{n}=1}^{\mathrm{N}}\left|\sin \left(\frac{\mathrm{n} \cdot \pi}{2}\right)\right| \cdot \frac{\sin \left(\mathrm{n} \cdot \frac{\gamma}{2}\right)}{\pi \cdot \mathrm{n}} \cdot \sin \left[(\mathrm{n}+1) \cdot \mathrm{w} \cdot \mathrm{t}-\mathrm{n} \cdot\left(\xi+\frac{\pi}{3}\right)+\frac{3 \cdot \pi}{2}\right]-2 \cdot \mathrm{V}_{\mathrm{p} 1} \cdot \sum_{\mathrm{n}=1}^{\mathrm{N}}\left|\sin \left(\frac{\mathrm{n} \cdot \pi}{2}\right)\right| \cdot \frac{\sin \left(\mathrm{n} \cdot \frac{\gamma}{2}\right)}{\pi \cdot \mathrm{n}} \cdot \sin \left[(\mathrm{n}-1) \cdot \mathrm{w} \cdot \mathrm{t}-\mathrm{n} \cdot\left(\xi+\frac{\pi}{3}\right)-\frac{3 \cdot \pi}{2}\right] \\
& \mathrm{V}_{\mathrm{oB}}(\mathrm{t}):=\mathrm{V}_{\mathrm{oB} 1}(\mathrm{t})-\mathrm{V}_{\mathrm{oB} 2}(\mathrm{t}) \\
& \mathrm{V}_{\mathrm{OY} l}(\mathrm{t}):=2 \cdot \mathrm{V}_{\mathrm{p}} \cdot \sum_{\mathrm{n}=1}^{\mathrm{N}}\left|\sin \left(\frac{\mathrm{n} \cdot \pi}{2}\right)\right| \cdot \frac{\sin \left(\mathrm{n} \cdot \delta_{1}\right)}{\mathrm{n} \cdot \pi} \cdot \sin \left[(\mathrm{n}+1) \cdot \mathrm{w} \cdot \mathrm{t}-\mathrm{n} \cdot \theta_{12}-\frac{2 \cdot \pi}{3}\right]-2 \cdot \mathrm{V}_{\mathrm{p}} \cdot \sum_{\mathrm{n}=1}^{\mathrm{N}}\left|\sin \left(\frac{\mathrm{n} \cdot \pi}{2}\right)\right| \cdot \frac{\sin \left(\mathrm{n} \cdot \delta_{1}\right)}{\mathrm{n} \cdot \pi} \cdot \sin \left[(\mathrm{n}-1) \cdot \mathrm{w} \cdot \mathrm{t}-\mathrm{n} \cdot \theta_{12}+\frac{2 \cdot \pi}{3}\right] \\
& \mathrm{V}_{\mathrm{OY} 2}(\mathrm{t}):=2 \cdot \mathrm{V}_{\mathrm{p} 1} \cdot \sum_{\mathrm{n}=1}^{\mathrm{N}}\left|\sin \left(\frac{\mathrm{n} \cdot \pi}{2}\right)\right| \cdot \frac{\sin \left(\mathrm{n} \cdot \frac{\gamma}{2}\right)}{\pi \cdot \mathrm{n}} \cdot \sin \left[(\mathrm{n}+1) \cdot \mathrm{w} \cdot \mathrm{t}-\mathrm{n} \cdot\left(\xi+\frac{2 \cdot \pi}{3}\right)+\frac{\pi}{6}\right]-2 \cdot \mathrm{V}_{\mathrm{p}} \cdot \sum_{\mathrm{n}=1}^{\mathrm{N}}\left|\sin \left(\frac{\mathrm{n} \cdot \pi}{2}\right)\right| \cdot \frac{\sin \left(\mathrm{n} \cdot \frac{\gamma}{2}\right)}{\pi \cdot \mathrm{n}} \cdot \sin \left[(\mathrm{n}-1) \cdot \mathrm{w} \cdot \mathrm{t}-\mathrm{n} \cdot\left(\xi+\frac{2 \cdot \pi}{3}\right)-\frac{\pi}{6}\right] \\
& \mathrm{V}_{\mathrm{OY}}(\mathrm{t}):=\mathrm{V}_{\mathrm{OY} 1}(\mathrm{t})+\mathrm{V}_{\mathrm{OY}} 2^{(\mathrm{t})} \\
& \mathrm{V}_{\mathrm{OR} 1}(\mathrm{t}):=2 \cdot \mathrm{V}_{\mathrm{p}} \cdot \sum_{\mathrm{n}=1}^{\mathrm{N}}\left|\sin \left(\frac{\mathrm{n} \cdot \pi}{2}\right)\right| \frac{\sin \left(\mathrm{n} \cdot \delta_{1}\right)}{\mathrm{n} \cdot \pi} \cdot \sin \left[(\mathrm{n}+1) \cdot \mathrm{w} \cdot \mathrm{t}-\mathrm{n} \cdot \theta_{11}\right]-2 \cdot \mathrm{V}_{\mathrm{p}} \cdot \sum_{\mathrm{n}=1}^{\mathrm{N}}\left|\sin \left(\frac{\mathrm{n} \cdot \pi}{2}\right)\right| \cdot \frac{\sin \left(\mathrm{n} \cdot \delta_{1}\right)}{\mathrm{n} \cdot \pi} \cdot \sin \left[(\mathrm{n}-1) \cdot \mathrm{w} \cdot \mathrm{t}-\mathrm{n} \cdot \theta_{11}\right] \\
& \mathrm{V}_{\mathrm{oR} 2}(\mathrm{t}):=2 \cdot \mathrm{V}_{\mathrm{p} 1} \cdot \sum_{\mathrm{n}=1}^{\mathrm{N}}\left|\sin \left(\frac{\mathrm{n} \cdot \pi}{2}\right)\right| \cdot \frac{\sin \left(\mathrm{n} \cdot \frac{\gamma}{2}\right)}{\pi \cdot \mathrm{n}} \cdot \sin \left[(\mathrm{n}+1) \cdot \mathrm{w} \cdot \mathrm{t}-\mathrm{n} \cdot \xi+\frac{5 \cdot \pi}{6}\right]-2 \cdot \mathrm{V}_{\mathrm{p} 1} \cdot \sum_{\mathrm{n}=1}^{\mathrm{N}}\left|\sin \left(\frac{\mathrm{n} \cdot \pi}{2}\right)\right| \cdot \frac{\sin \left(\mathrm{n} \cdot \frac{\gamma}{2}\right)}{\pi \cdot \mathrm{n}} \cdot \sin \left[(\mathrm{n}-1) \cdot \mathrm{w} \cdot \mathrm{t}-\mathrm{n} \cdot \xi-\frac{5 \cdot \pi}{6}\right] \\
& \mathrm{V}_{\mathrm{oR}}(\mathrm{t}):=\mathrm{V}_{\mathrm{OR} 1}(\mathrm{t})+\mathrm{V}_{\mathrm{OR} 2}(\mathrm{t}) \\
& \mathrm{U}_{\mathrm{o}}(\mathrm{t}):=\mathrm{V}_{\mathrm{OR}}(\mathrm{t})+\mathrm{V}_{\mathrm{OY}}(\mathrm{t})+\mathrm{V}_{\mathrm{OB}}(\mathrm{t})
\end{aligned}
$$


$\mathrm{n}:=1$

$$
\begin{aligned}
& \mathrm{V}_{\mathrm{B} 1}:=0-\left[2 \cdot \mathrm{V}_{\mathrm{p}} \cdot\left(\frac{\sin \left(\mathrm{n} \cdot \delta_{1}\right)}{\mathrm{n} \cdot \pi} \cdot \sin \left(0-\mathrm{n} \cdot \theta_{13}-\frac{2 \cdot \pi}{3}\right)\right)\right]+\left[0-2 \cdot \mathrm{V}_{\mathrm{p} 1} \cdot\left[\frac{\sin \left(\mathrm{n} \cdot \frac{\gamma}{2}\right)}{\pi \cdot \mathrm{n}} \cdot \sin \left[0-\mathrm{n} \cdot\left(\xi+\frac{\pi}{3}\right)-\frac{3 \cdot \pi}{2}\right]\right]\right] \\
& \left.\mathrm{V}_{\mathrm{Y} 1}:=0-2 \cdot \mathrm{V}_{\mathrm{p}} \cdot\left(\frac{\sin \left(\mathrm{n} \cdot \delta_{1}\right)}{\mathrm{n} \cdot \pi} \cdot \sin \left(0-\mathrm{n} \cdot \theta_{12}+\frac{2 \cdot \pi}{3}\right)\right)+\left[0-2 \cdot \mathrm{V}_{\mathrm{p} 1} \cdot\left[\frac{\sin \left(\mathrm{n} \cdot \frac{\gamma}{2}\right)}{\pi \cdot \mathrm{n}} \cdot \sin \left[0-\mathrm{n} \cdot\left(\xi+\frac{2 \cdot \pi}{3}\right)-\frac{\pi}{6}\right]\right]\right]\right] \\
& \mathrm{V}_{\mathrm{R} 1}:=0-2 \cdot \mathrm{V}_{\mathrm{p}} \cdot\left(\frac{\sin \left(\mathrm{n} \cdot \delta_{1}\right)}{\mathrm{n} \cdot \pi} \cdot \sin \left(0-\mathrm{n} \cdot \theta_{11}\right)\right)-2 \cdot\left[\mathrm{V}_{\mathrm{p} 1} \cdot\left(\frac{\sin \left(\mathrm{n} \cdot \frac{\gamma}{2}\right)}{\pi \cdot \mathrm{n}} \cdot \sin \left(0-\mathrm{n} \cdot \xi-\frac{5 \cdot \pi}{6}\right)\right)\right] \\
& \mathrm{V}_{\mathrm{dco}}:=\mathrm{V}_{\mathrm{R} 1}+\mathrm{V}_{\mathrm{Y} 1}+\mathrm{V}_{\mathrm{B} 1}
\end{aligned}
$$

\section{Appendix C: MathCAD Current Equations}

Output current expanded expression

$$
\begin{aligned}
& \text { OUTPUT CURRENT }
\end{aligned}
$$

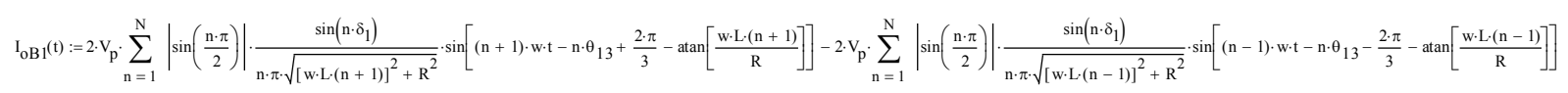

$$
\begin{aligned}
& \mathrm{I}_{\mathrm{OB} 2}(\mathrm{t}):=2 \cdot \mathrm{V}_{\mathrm{p} 1} \cdot \sum_{\mathrm{n}=1}^{\mathrm{N}}\left|\sin \left(\frac{\mathrm{n} \cdot \pi}{2}\right)\right| \cdot \frac{\sin \left(\mathrm{n} \cdot \frac{\gamma}{2}\right)}{\pi \cdot \mathrm{n} \cdot \sqrt{[\mathrm{w} \cdot \mathrm{L} \cdot(\mathrm{n}+1)]^{2}+\mathrm{R}^{2}}} \cdot \sin \left[(\mathrm{n}+1) \cdot \mathrm{w} \cdot \mathrm{t}-\mathrm{n} \cdot\left(\xi+\frac{\pi}{3}\right)+\frac{3 \cdot \pi}{2}-\operatorname{atan}\left[\frac{\mathrm{w} \cdot \mathrm{L} \cdot(\mathrm{n}+1)}{\mathrm{R}}\right]\right]-2 \cdot \mathrm{V}_{\mathrm{p} 1} \cdot \sum_{\mathrm{n}=1}^{\mathrm{N}}\left|\sin \left(\frac{\mathrm{n} \cdot \pi}{2}\right)\right| \cdot \frac{\sin \left(\mathrm{n} \cdot \frac{\gamma}{2}\right)}{\pi \cdot \mathrm{n} \cdot \sqrt{[\mathrm{w} \cdot \mathrm{L} \cdot(\mathrm{n}-1)]^{2}+\mathrm{R}^{2}}} \cdot \sin \left[(\mathrm{n}-1) \cdot \mathrm{w} \cdot \mathrm{t}-\mathrm{n} \cdot\left(\xi+\frac{\pi}{3}\right)-\frac{3 \cdot \pi}{2}-\operatorname{atan}\left[\frac{\mathrm{w} \cdot \mathrm{L} \cdot(\mathrm{n}-1)}{\mathrm{R}}\right]\right] \\
& \mathrm{I}_{\mathrm{OB}}(\mathrm{t}):=\mathrm{I}_{\mathrm{OB}}\left(\mathrm{t}^{(\mathrm{t})}-\mathrm{I}_{\mathrm{OB2}} \mathrm{C}^{(\mathrm{t})}\right.
\end{aligned}
$$

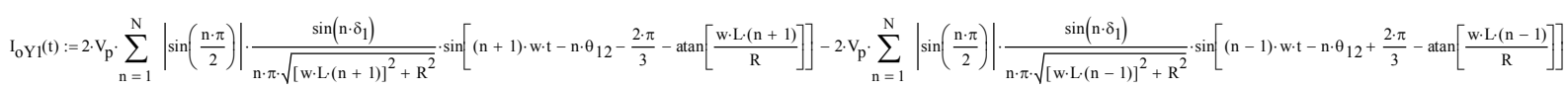

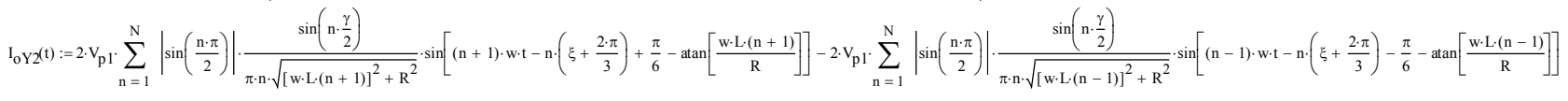

$$
\begin{aligned}
& \mathrm{I}_{\mathrm{o}} \mathrm{Y}^{(\mathrm{t})}:=\mathrm{I}_{\mathrm{OY}}(\mathrm{t})+\mathrm{I}_{\mathrm{OY}}(\mathrm{t}) \\
& I_{0 R I}(t):=2 \cdot V_{p} \cdot \sum_{n=1}^{N}\left|\sin \left(\frac{n \cdot \pi}{2}\right)\right| \cdot \frac{\sin \left(n \cdot \delta_{1}\right)}{n \cdot \pi \cdot \sqrt{[w \cdot L \cdot(n+1)]^{2}+R^{2}}} \cdot \sin \left[(n+1) \cdot w \cdot t-n \cdot \theta_{11}-\operatorname{atan}\left[\frac{w \cdot L \cdot(n+1)}{R}\right]\right]-2 \cdot V_{p} \cdot \sum_{n=1}^{N}\left|\sin \left(\frac{n \cdot \pi}{2}\right)\right| \cdot \frac{\sin \left(n \cdot \delta_{1}\right)}{n \cdot \pi \cdot \sqrt{[w \cdot L \cdot L \cdot(n-1)]^{2}+R^{2}}} \cdot \sin \left[(n-1) \cdot w \cdot t-\left[n \cdot \theta_{11}+\operatorname{atan}\left[\frac{w \cdot L \cdot(n-1)}{R}\right]\right]\right] \\
& \mathrm{I}_{\mathrm{oR} 2}(\mathrm{t})=2 \cdot \mathrm{v}_{\mathrm{p} 1} \cdot \sum_{\mathrm{n}=1}^{\mathrm{N}}\left|\sin \left(\frac{\mathrm{n} \cdot \pi}{2}\right)\right| \frac{\sin \left(\mathrm{n} \cdot \frac{\gamma}{2}\right)}{\pi \cdot \mathrm{n} \cdot \sqrt{[\mathrm{w} \cdot \mathrm{L} \cdot(\mathrm{n}+1)]^{2}+\mathrm{R}^{2}}} \cdot \sin \left[(\mathrm{n}+1) \cdot \mathrm{w} \cdot \mathrm{t}-\mathrm{n} \cdot \xi+\frac{5 \cdot \pi}{6}-\operatorname{atan}\left[\frac{\mathrm{w} \cdot \mathrm{L}(\mathrm{n}+1)}{\mathrm{R}}\right]\right]-2 \cdot \mathrm{v}_{\mathrm{p} 1} \cdot \sum_{\mathrm{n}=1}^{\mathrm{N}}\left|\sin \left(\frac{\mathrm{n} \cdot \pi}{2}\right)\right| \frac{\sin \left(\mathrm{n} \cdot \frac{\gamma}{2}\right)}{\pi \cdot \mathrm{n} \cdot \sqrt{[\mathrm{w} \cdot \mathrm{L} \cdot(\mathrm{n}-1)]^{2}+\mathrm{R}^{2}}} \cdot \sin \left[(\mathrm{n}-1) \cdot \mathrm{w} \cdot \mathrm{t}-\mathrm{n} \cdot \xi-\frac{5 \cdot \pi}{6}-\operatorname{atan}\left[\frac{\mathrm{w} \cdot \mathrm{L} \cdot(\mathrm{n}-1)}{\mathrm{R}}\right]\right] \\
& \mathrm{I}_{\mathrm{OR}}(\mathrm{t}):=\mathrm{I}_{\mathrm{OR}} \mathrm{I}^{(\mathrm{t})}+\mathrm{I}_{\mathrm{OR}} 2^{(t)} \\
& \mathrm{I}_{\mathrm{EO}}(\mathrm{t}):=\mathrm{I}_{\mathrm{OR}}(\mathrm{t})+\mathrm{I}_{\mathrm{O}} \mathrm{Y}^{(\mathrm{t})}+\mathrm{I}_{\mathrm{OB}} \mathrm{B}^{(t)}
\end{aligned}
$$

